\title{
Policy-Based QoS-Aware Packet Scheduling for CDMA 1x Ev-DO
}

\author{
Jinho Hwang ${ }^{1}$, M. Tamer Refaei ${ }^{2}$, Hyeong-Ah Choi ${ }^{1}$, Jae-Hoon Kim ${ }^{3}$, JungKyo Sohn ${ }^{4}$, Hyeong In Choi ${ }^{4}$ \\ ${ }^{1}$ Department of Computer Science, George Washington University, Washington, DC \\ ${ }^{2}$ Bradley Department of Electrical and Computer Engineering, Virginia Tech, Alexandria, VA \\ ${ }^{3}$ Access Network \& Mobile Terminal R\&D Center, SK Telecom, Seoul, Korea \\ ${ }^{4}$ Department of Mathematics, Seoul National University, Seoul, Korea \\ \{jhhwang,hchoi\}@gwu.edu, mtamer@vt.edu, jayhoon.kim@gmail.com, \{jgsohn,hichoi\}@snu.ac.kr
}

\begin{abstract}
CDMA 1x Ev-DO is an evolution of the CDMA2000 3G wireless standard to enable high rate packet data services up to $2.4 \mathrm{Mbps}$ in $\operatorname{Rev} 0,3.1 \mathrm{Mbps}$ in Rev $A$, and 4.9Mbps in Rev B. To support such high data rates, CDMA 1x Ev-DO utilizes TDMA technology on the downlink allowing a single receiver per time slot. Accordingly, a scheduling algorithm is necessary to determine which user receives data in a given time slot. Typically, schedulers consider efficiency (increasing network throughput) or fairness (fair allocation of resources among users) as the basis for scheduling. To realize a reasonable tradeoff between efficiency and fairness, opportunistic schedulers have been proposed that take advantage of instantaneous improvements in users radio conditions by serving them at higher rates. However, given the increasing demand to support user QoS requirements, a QoS-aware scheduler may need to serve users even at times of inadequate radio conditions. In this paper, we propose a QoS-aware packet scheduling algorithm that takes into account policy rules that govern the relationships between different user QoS classes. Our scheduler uses marginal utility functions defined to embody the given rules when selecting users to be served on the downlink. Our scheduling algorithm is implemented in the OPNET module of the CDMA 1x EvDO system that we have developed, and its performance is compared to other scheduling algorithms. The simulation results show that our scheduler performs well ensuring the policy rules are followed, meeting users' QoS requirements, and providing fairness among users within the same QoS class.
\end{abstract}

Keywords: CDMA $1 x$ Ev-DO, 3GPP2, Wireless Networks, Packet Scheduling, Quality of Service

\section{INTRODUCTION}

The advent of $3 \mathrm{G}$ cellular networks has enabled cellular networks to support high rate data services. CDMA 1x Ev-DO (HDR), an evolution of the CDMA2000 3G wireless standard, is a technology that is optimized for high rate packet data services. CDMA 1x Ev-DO enables up to $2.4 \mathrm{Mbps}$ in $\operatorname{Rev} 0$ [1], which is increased to 3.1Mbps in Rev A [2] and to 4.9Mbps in Rev B [3].

This work was in part supported by the Access Network \& Mobile Terminal R\&D Center, SK Telecom, Seoul, Korea.
To support such high data rates, CDMA 1x Ev-DO utilizes TDMA technology on the downlink (as opposed to CDMA in IS-95 and CDMA2000) allowing a single receiver per time slot. The size of each slot is $1.67 \mathrm{~ms}$ making it short enough so that the channel quality of a user stays approximately constant within one or even a few consecutive time slots [18]. Some of the key features of CDMA 1x Ev-DO are:

- Only data is supported in CDMA 1x Ev-DO (i.e. no support for voice).

- Power control is no longer an issue for the downlink as compared to CDMA2000 since only a single user is being served per time slot. Rather, rate control is a more important issue.

- Some radio resource management (RRM) functions moved from the BSC (Base Station Controller) to the BTS (Base Transceiver Subsystem), and new ones were added such as packet scheduling, AMC (Adaptive Modulation Coding), and HARQ (Hybrid Automatic Repeat Request).

Packet scheduling is one of the key components of CDMA 1x Ev-DO. The design of scheduling algorithms for mobile communication networks is especially challenging given the highly variable link error rates and capacities, and the changing mobile station connectivity typically encountered in such networks [7]. Moreover, the emergence of new applications with diverse QoS constraints for data services such as throughput, delay, jitter, or loss constraints impose another challenge on scheduling algorithms, which are expected to consider and honor such requirements. Conventional downlink scheduling algorithms in CDMA $1 \mathrm{x}$ Ev-DO either focus on increasing network throughput, such as Max C/I, fairness (equal allocation of network resources among all users), such as Round Robin, or a tradeoff of both such as Proportional Fair. However, none of the three consider users' QoS requirements as one of the scheduling criteria. 
In this paper, our objective is to develop a policybased QoS-aware packet scheduling algorithm. Given the guaranteed throughput as a QoS requirement for each user class, we define a set of policy rules that govern the relationships between the different throughput-based QoS classes offered by a cellular operator. The rules ensure that users of a higher QoS class are always prioritized higher to meet their throughput requirements compared to users of a lower class as long as it is possible. They also ensure that fairness is provided among users within the same QoS class. These rules are fed into the packet scheduler which follows the policy rules while scheduling users for downlink transmissions. We then develop a complete CDMA 1x Ev-DO system in OPNET and evaluate the effectiveness of our scheduling algorithm by comparing it against others. Our results show that our scheduler performs well ensuring the policy rules are followed, meeting user's QoS requirements, and providing fairness among users within the same QoS class.

Our contribution in this paper is three fold. Firstly, we present a guideline for designing marginal utility functions for user classes that embody the given arbitrary policy rules that govern the relationships between different user QoS classes. Secondly, we develop a scheduler using the marginal utility functions that follows the rules when selecting users to be served. Thirdly, we develop the CDMA 1x Ev-DO system in the OPNET simulator and utilize our implementation to evaluate the effectiveness of our scheduling algorithm.

\section{A. Background}

Conventional packet scheduling algorithms for CDMA 1x Ev-DO are Round Robin, Max C/I, and Proportional Fair [24]. Round Robin scheduling algorithm serves one user at a time in a round robin format. This ignores network efficiency as some of the users may be experiencing inadequate radio conditions, which reduces the overall network throughput. Max C/I on the other hand focuses on efficiency by serving the user with the highest $\mathrm{C} / \mathrm{I}$. This achieves the maximum network throughput (since the user with the Max C/I will be offered a high data rate) but reduces fairness among users due to the possibility that this user (i.e. the one with the highest $\mathrm{C} / \mathrm{I}$ ) will monopolize the network resources as he will be selected by the scheduler at all times. Proportional fairness algorithm realizes the tradeoff between efficiency and fairness by considering both, users' $\mathrm{C} / \mathrm{I}$ as well as throughput received, as the selection criteria of the scheduler. An increase in a user's
$\mathrm{C} / \mathrm{I}$ is taken advantage of by serving him at a higher rate. On the other hand, the likelihood of a user being selected increases as its received throughput decreases. In [25], the authors conduct a performance comparison between the three scheduling algorithms, Round Robin, Max C/I, and Proportional Fair.

Realizing the need for schedulers that can respond to the increasing demand to support user QoS requirements, several scheduling algorithms have been proposed that are QoS aware. In [18], a Longest Weighted Delay First (M-LWDF) scheduling algorithm is described. The algorithm is throughput optimal in the sense that it is able to keep all user queues from overflowing. The algorithm was adapted to be QoS aware providing users with their minimum throughput guarantees. In [13], a Weighted Fair Queueing based Proportional Fair algorithm is proposed. The algorithm is meant to provide equal fairness among users according to their position. In [19], an adaptive scheduling methodology is proposed to support the maximum possible throughput for NRT (Non Real Time) users while satisfying the QoS requirements of all ART (Almost Real Time) in an environment of mixed user services. In [20], a fair scheduling algorithm considering QoS demands was proposed. The algorithm was evaluated based on a simple OPNET implementation using a single link. In [15], the author considered QoS requirements in a WCDMA system. Two user QoS classes based on throughput are considered and are evaluated, but the proposed approach cannot be easily generalized to accommodate more than two classes or satisfy different rules or policies.

\section{B. Structure of Paper}

This paper is organized as follows. In section II, we discuss the the system architecture. In section III we formulate and describe the problem considered in this paper including the QoS policy rules as well as the scheduling algorithm. In section 3, we discuss the CDMA 1x EvDO simulator that we developed under OPNET and use it to evaluate our scheduler. We conclude in section IV.

\section{System Architecture}

Typically, a CDMA 1x Ev-DO network is composed of a BSC (Base Station Controller) that is connected to and is in control of admission control to a number of BTS (Base Transceiver Subsystem). Each user is associated with a BTS. All users in the network are mobile with varying speeds. All mobile user equipments have the same capabilities. 
TABLE I

PRoperties of THE PACKETS IN DOWNLINK CORRESPONDING TO DRC IN REVISION 0

\begin{tabular}{|l||l|l|l|l|l|l|}
\hline \hline DRC & DataRate $($ kbps $)$ & Slots & PhysicalPacketSize & FEC & Modulation & Ec $/ N t(d B)$ \\
\hline \hline 0x1 & 38.4 & 16 & 1024 & $1 / 5$ & QPSK & -11.5 \\
\hline 0x2 & 76.8 & 8 & 1024 & $1 / 5$ & QPSK & -9.7 \\
\hline 0x3 & 153.6 & 4 & 1024 & $1 / 5$ & QPSK & -6.8 \\
\hline 0x4 & 307.2 & 2 & 1024 & $1 / 5$ & QPSK & -3.9 \\
\hline 0x5 & 307.2 & 4 & 2048 & $1 / 3$ & QPSK & -3.8 \\
\hline 0x6 & 614.4 & 1 & 1024 & $1 / 3$ & QPSK & -0.8 \\
\hline 0x7 & 614.4 & 2 & 2048 & $1 / 3$ & QPSK & -0.6 \\
\hline 0x8 & 921.6 & 2 & 3072 & $1 / 3$ & 8-PSK & 1.8 \\
\hline 0x9 & $1,228.8$ & 1 & 2048 & $1 / 3$ & 16 QAM & 3.7 \\
\hline 0xa & $1,228.8$ & 2 & 4096 & $1 / 3$ & 16 QAM & 3.8 \\
\hline 0xb & $1,843.2$ & 1 & 3072 & $1 / 3$ & 8-PSK & 7.5 \\
\hline 0xc & $2,457.6$ & 1 & 4096 & $1 / 3$ & 16 QAM & 9.7 \\
\hline
\end{tabular}

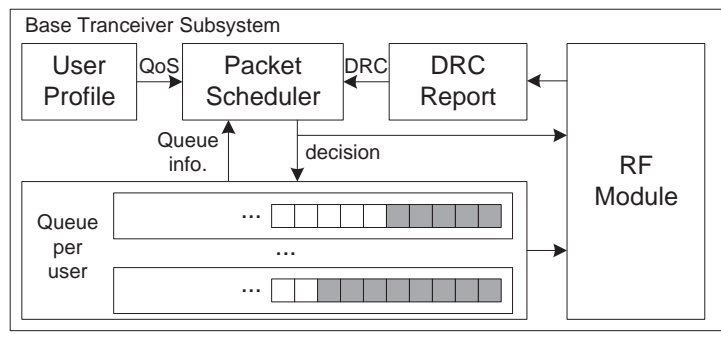

Fig. 1. BTS Functional Architecture

As mentioned before, some of the functionalities that typically resided in the BSC in IS-95 and CDMA2000 moved to the BTS in CDMA 1x Ev-DO such as packet scheduling. Since we are concerned with packet scheduling, our focus is on the BTS. Figure 1 shows the functional architecture of the BTS. The RF Module consists of a transmitter and a receiver for communication between the BTS and mobile stations. The DRC (Data Rate Control) report is a module that is responsible for receiving and processing information about channel conditions at each mobile station. As mentioned above, power control is not an issue in CDMA $1 \mathrm{x}$ Ev-DO based systems since they employ a TDM-based scheme for the downlink. Rather, rate control is a more important issue. In order to transmit on the downlink to a particular receiving mobile station at an appropriate data rate, the BTS needs an estimate of the channel conditions at that receiver. CDMA 1x Ev-DO employs a feedback mechanism whereby each mobile station measures the received signal strength from a pilot channel sent by the BTS and report back the measurement to the BTS. The feedback is in the form a DRC index, which reflects the maximum data rate the mobile station can receive given its surrounding channel conditions. Accordingly, the BTS adapts the data rate to be sent to the user by controlling physical layer properties such as coding rate, modulation, etc. Table 1 shows different values of DRC index and their corresponding data rates [1]. (Similar values are also available for Rev. A \& B.) The DRC index may then be used by the packet scheduling component as a user selection criterion. User Profile is another component within the BTS. This component has knowledge of user QoS requirements and is fed into the scheduler which is expected to consider user QoS requirements. The final component is for queuing which maintains a separate data queue for each user per QoS class.

\section{Problem Formulation AND SCHEDUling}

Consider a cellular operator with a set $N$ of mobile users. The operator offers its users $m$ different service classes that differ amongst each other based on the minimum guaranteed throughput.

Each mobile station $x \in N$ sends its DRC reading $D R C_{x}(t)$ to the BTS at each time slot $t$. The scheduling algorithm then selects a mobile station $y \in N$ to be receiving data in the current time slot $t$. Based on its $D R C_{y}(t)$ reading, the BTS assigns the mobile station $y$ the data rate $d_{y}(t)$ that corresponds to its $D R C_{y}(t)$. Let $t h_{x}(t)$ be a moving average of the throughput received by a mobile node $x$ at time slot $t$ over the most recent $w_{0}$ time slots. We define $t h_{x}(t)$ as:

- If user $x$ has been in the system for at least $w_{0}$ time slots,

$t h_{x}(t)=t h_{x}(t-1)+\frac{d_{x}(t) h_{x}(t)}{w_{0}}-\frac{t h_{x}(t-1)}{w_{0}}$

- If user $x$ entered the system in time slot $s_{x}$ where 


$$
\begin{aligned}
\left(t-s_{x}+1\right) & <w_{0}, \\
t h_{x}(t) & =\frac{\sum_{j=0}^{s_{x}} d_{x}(t-j) h_{x}(t-j)}{w_{0}}
\end{aligned}
$$

where $h_{x}(t)=1$ if user $x$ is scheduled in time $t$, and $h_{x}(t)=0$ otherwise.

\section{A. Bandwidth Allocation Policy}

We now introduce our policy that recognizes and draws the line between different user throughput-based QoS classes. The policy defines rules that govern the relationship between different user classes. Let $C(x)$ denotes the service class which user $x$ belongs to where $C(y)<C(x)$ means that QoS class $C(x)$ has higher preference than $C(y)$. Note that users in the same QoS class have the same guaranteed (or minimum) throughput. Let $B(x)$ denote the guaranteed bit rate or the guaranteed throughput of user $x$. Assume that $B(x)=B(y)$ if $C(x)=C(y)$. For any two users $x, y \in N$ the policy rules are defined as follows.

- Policy Rule 1: In any time slot $t$, user $x \in N$ with throughput $t h_{x}(t)<B(x)$ has a higher scheduling priority than any other user $y \in N$ whose QoS class $C(y)<C(x)$. Hence, a user $y \in N$ can be scheduled if and only if all users $x \in N$, where $C(y)<C(x)$, have their throughput $t h_{x}(t) \geq B(x)$.

- Policy Rule 2: In any time slot $t$, user $x \in N$ with $t h_{x}(t) \geq B(x)$ has a lower scheduling priority than any other user $y \in N$ whose service class $C(y)<C(x)$ with $t h_{y}(t)<B(y)$. This means that once the throughput guarantees of all users in a particular service class are satisfied, the scheduling priority shifts to users of the lower service classes.

- Policy Rule 3: In any time slot $t$, if all users meet their guaranteed throughput, the remaining capacity must be distributed such that for any two users $x, y \in N$, the throughput ratio $t h_{x}(t) / t h_{y}(t)=\alpha_{C(x), C(y)}$, where $\alpha_{C(x), C(y)}$ is an arbitrary number given as a part of the policy rules.

Note that if the available data rate $d_{x}(t)$ corresponding to $D R C_{x}(t)$ of user $x$ is always or mostly less than the guaranteed minimum throughput $B(x)$ due to his bad signal quality, the throughput of user $x$ will never reach the minimum value $B(x)$. Hence, it becomes difficult to satisfy the QoS requirements of such a user, and we will simply assume that this extreme case does not exist in our discussion.

\section{B. Scheduling Algorithm}

Our scheduling algorithm is based on marginal utility functions (that will be discussed in the next section) embodying the given bandwidth allocation policy rules. Let $f_{C(x)}\left(t h_{x}(t)\right)$ denote a marginal utility function defined for the service class $C(x)$ taking the current throughput $t h_{x}(t)$ of a user $x$ in class $C(x)$ as the input.

In each time slot $t$, the scheduling algorithm selects and schedules user $x *$ that satisfies the following:

$$
x *=\arg \max _{x \in N}\left\{f_{C(x)}\left(t h_{x}(t)\right) \cdot d_{x}(t)\right\} .
$$

Accordingly, $h_{x}(t)$ is set to 1 for the selected user and to 0 for all other users. The throughput $t h_{x}(t), \forall x \in$ $N$, is updated as shown in Equations (1) and (2).

\section{Marginal Utility Function}

Consider $k$ different user classes $C_{1}, \cdots, C_{k}$ such that (i) the guaranteed minimum throughput rate for users in class $C_{i}$ is denoted by $B_{i}$, where $B_{1}<\cdots<B_{k}$ and (ii) a preference order that is independent from the minimum throughput requirement is given to the $k$ classes, i.e., $B_{i}<B_{j}$ for some $1 \leq i, j \leq k$, but $C_{i}$ may have a higher preference than $C_{j}$. Let $D=\left\{d_{1}, \cdots, d_{m}\right\}$ denote the set of available data rate corresponding to the DRC index in CDMA 1x Ev-DO system such that $d_{1}=d_{\min }<d_{2}<\cdots<d_{m}=d_{\max }$. In Revision 0, for example, 12 different DRC indices exist as shown in Table I where $d_{\min }=38.4 \mathrm{kbps}$ and $d_{\max }=2,457.6 \mathrm{kbps}$.

Let $C_{i}$ and $C_{j}$ be arbitrary user classes with $B_{i}<$ $B_{j}$. Assuming that $C_{j}$ has a higher preference than $C_{i}$, we define marginal utility functions $f_{j}$ and $f_{i}$ for classes $C_{i}$ and $C_{j}$, respectively, such that:

$$
\begin{gathered}
f_{j}(b)= \begin{cases}z_{j} & \text { if } 0 \leq b<B_{j} \\
-b+d_{\max } & \text { if } B_{j} \leq b \leq d_{\text {max }}\end{cases} \\
f_{i}(b)= \begin{cases}z_{i} & \text { if } 0 \leq b<B_{i} \\
-\alpha_{C_{j}, C_{i}} b+d_{\max } & \text { if } B_{i} \leq b \leq d_{\text {max }}\end{cases}
\end{gathered}
$$

where $z_{j}$ and $z_{i}$ are constants defined such that $z_{j} d_{\min }>$ $z_{i} d_{\max }$ so that Rule 1 is satisfied even if a user in class $C_{j}$ has a low DRC index. Similarly, $f_{j}\left(B_{j}\right)$ is defined such that $z_{i} d_{\min }>f_{j}\left(B_{j}\right) d_{\max }$ to satisfy Rule 2 . Suppose the current throughput of a user $x$ in class $C_{i}$ and that of a user $y$ in class $C_{j}$ are $b_{0}$ and $\alpha b_{0}$ respectively, where $\alpha=\alpha_{C_{j}, C_{i}}$. Then, $f_{i}\left(b_{0}\right)=f_{j}\left(\alpha b_{0}\right)$ 
as shown in Figure 2; hence, if DRC indices are same for $x$ and $y$, then Rule 3 will also be satisfied.

When $C_{i}$ has a higher preference than $C_{j}, f_{i}$ and $f_{j}$ can be similarly constructed with minor changes.

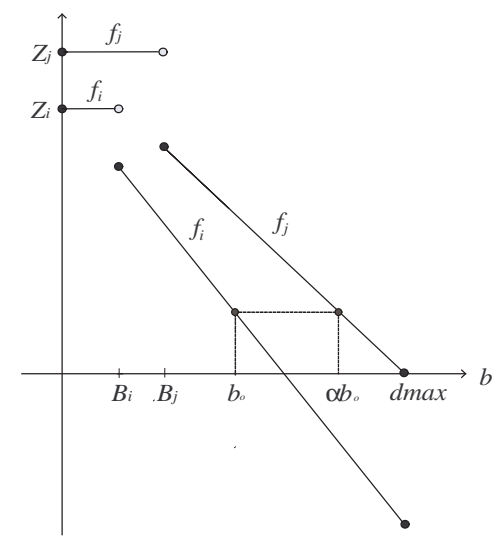

Fig. 2. Marginal Utility Function: $f_{j}=-b+d_{\max }$ and $f_{i}=$ $-\alpha b+d_{\max }$

\section{Simulation}

In this section we describe the CDMA $1 \mathrm{x}$ EvDO simulator that we developed using OPNET and utilize the simulator to evaluate the performance of our scheduling algorithm.

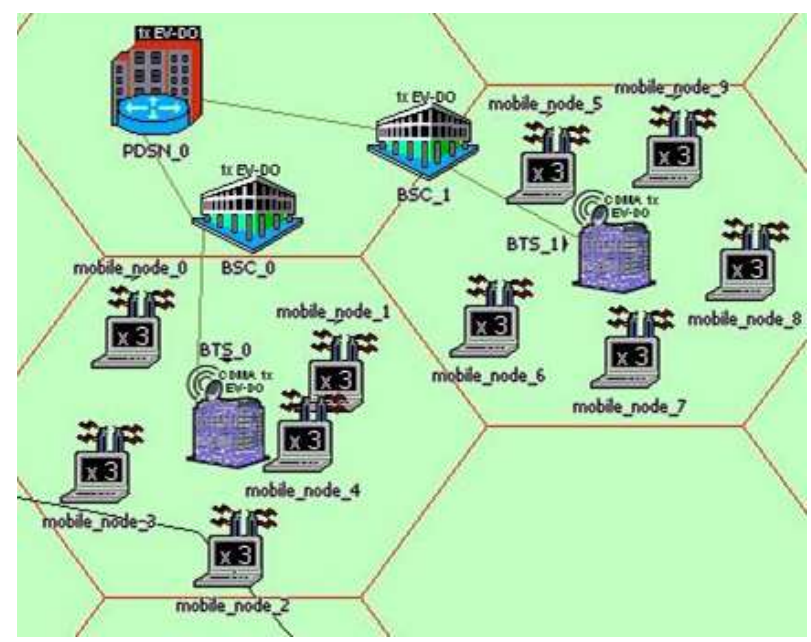

Fig. 3. OPNET Scenario: one PDSN, two BSCs each attached to one BTS. Each BTS has 5 users connected to it.

\section{A. OPNET Simulator}

We developed the CDMA 1x Ev-DO system under OPNET simulator. Figure 3 shows a snapshot of an ex- ample CDMA 1x Ev-DO network topology. The system considers a single downlink channel with slot length of $1.67 \mathrm{msec}$. A single queue is maintained at the BTS for each user. Each Queue holds data packets waiting to be sent on the downlink to its corresponding user. Each time slot the packet scheduler at the BTS selects a user (which already has data packets queued) to be receiving data. Based on the scheduling criterion shown in Equation (3), a user is selected. Depending on the selected user's data rate, the system may choose to encapsulate one or more data packets from his queue within the RLC/MAC frame to meet the required data rate.

\section{B. Scheduler Evaluation}

We consider a network environment with two BTS connected to a single BSC. The network supports data rates in $R=\{38.4,76.8,153.6,307.2,614.4,921.6$, $1228.8,1843.2,2457.6\}$. Hence, $r_{\min }=38.4$ and $r_{\max }=2457.6$. We consider two QoS classes Gold and Silver. The Gold QoS class guarantees a minimum throughput of $128 \mathrm{Kbps}$ while the Silver QoS class guarantees a minimum throughput of $64 \mathrm{Kbps}$.

We assume that the Gold class has a higher preference than the Silver class, and $\alpha_{\text {Gold, Silver }}=2$, i.e., the bandwidth after satisfying the minimum throughput requirement of each user is distributed such that the throughput of the Gold class users should be twice of that of Silver class users. The marginal utility functions $f_{\text {Gold }}$ and $f_{\text {Silver }}$ are then defined as discussed earlier. We evaluate the following aspects of our scheduling algorithms:

- QoS Awareness: In the presence of users from both QoS classes (i.e. Gold and Silver), we evaluate the system to assure that the policy rules are in effect. Firstly, we evaluate the ability of the scheduler to provide users of each QoS class with their guaranteed throughout under normal conditions. Secondly, we ensure the higher preference of the Gold class to that of the Silver class in overloaded conditions. Thirdly, we consider the impact of emergence of Gold class users on the throughput of the Silver class users and vice versa. In each case, we consider the average throughput of all users from each QoS class as our metric for evaluating the QoS awareness of the scheduler. We calculate the average throughput attained by users of the Gold QoS class and compare against that of users from the Silver QoS class. We also compare against Max C/I, Round Robin, and Proportional Fair scheduling algorithms, which do not consider user QoS requirements (i.e. QoS unaware). 
- Fairness: A fair scheduler should provide fairness among users of the same QoS class. We evaluate the fairness of our scheduler compared to other schedulers. We consider the standard deviation in the throughput of users of each class as our evaluation metric for a scheduler's fairness.

1) Scenario 1: Firstly, to evaluate the QoS awareness of our scheduler, we use a simulation scenario with 12 Gold users and 12 Silver users. All 24 users are connected to a BTS $A$. A second BTS $B$ exist in the network and is connected to BTS $A$ through a BSC. Another set of 24 users are connected to BTS $B$ that act only as traffic sources. Each of the 24 users connected to BTS $B$ generates enough traffic so that there is always data available in the queue of each Gold or Silver user connected to BTS $A$. No mobility is adopted for users in this scenario and all users are placed within close range of the BTS (i.e. good channel conditions). We evaluate the average throughput of Gold and Silver using four different scheduling algorithm: Round Robin (RR), Max C/I (Max), Proportional Fair (PF), and our algorithm (Policy).

Figure 4 shows results for the above simulation scenario. As shown, the average throughput of Gold and Silver users is the same for all three schedulers that are QoS unaware (i.e. RR, Max, and PF). None of the three schedulers differentiate between users since none of them consider user QoS class as a scheduling criterion. On the other hand, our scheduler recognizes the difference in QoS requirements between Gold and Silver. Both the Gold and Silver users meet their QoS requirements (since enough network resources are available to do so). The average throughput of a Gold user is about $135 \mathrm{Kbps}$ while that of a Silver user is about $68 \mathrm{Kbps}$, which means that the system distributes the excess throughput offered for users of both classes in proportion to their QoS class as directed by rule 3 .

2) Scenario 2: We use a second simulation scenario to evaluate our scheduler under network overload situations. In this simulation scenario, we increase the number of Gold and Silver users connected to BTS $A$ to 15 each. We also increase the traffic sources connected to BTS $B$ to 30 . In this scenario, the 15 traffic sources whose traffic is destined to Gold users generate more traffic compared the 15 traffic sources whose traffic is destined to Silver users. The combined traffic load generated by the 30 traffic source users exceeds the capacity of the network (capacity of the network is $2.4 \mathrm{Mbps}$ based on CDMA 1x Ev-DO Rev. 0). We evaluate the performance of our scheduler under such scenario and compare against QoS unaware schedulers. As shown in Figure 5, the throughput of Gold users is maintained at its guaranteed throughput $(128 \mathrm{Kbps})$ on the expense of reducing the throughput of Silver users $(23 \mathrm{Kbps})$ since Gold users have a higher preference. Other schedulers that are QoS unaware reduce the throughput of the Gold users so that it is close to that of the Silver users (RR) or reduce the throughput of both the Gold and Silver users (Max C/I and PF). Using Round Robin, the average throughput of Gold users peeks at $77 \mathrm{Kbps}$ while that of the Silver users peeks at $64 \mathrm{Kbps}$. The average throughput of Gold users peeks at $76 \mathrm{Kbps}$ while that of the Silver users peeks at $32 \mathrm{Kbps}$ using Max C/I. With respect to Proportional Fair, the average throughput of Gold users peeks at $77 \mathrm{Kbps}$ while that of the Silver users peeks at $17 \mathrm{Kbps}$. None of the schedulers meet the QoS requirements of the Gold user. Our scheduler places the Gold user at a higher scheduling priority compared to the Silver users meeting its QoS requirements first.

3) Scenario 3: We use a third simulation scenario that is a variant of the first simulation scenario to evaluate the impact of emergence of Gold users on the throughput of Silver users. Using 9 Gold users and 12 Silver users that are not mobile and are positioned within close range of the BTS (i.e. good channel conditions). A set of three Gold users are initially placed outside of range of BTS $A$ are moving to the range of BTS $A$ after 10 seconds from the simulation start time. The rest of the traffic sources connected to BTS $B$ remain the same as in the first simulation scenario. As shown in Figure 6, as soon as the three Gold users become within range, the scheduling priority shifts fast to attempt to provide them with their guaranteed throughput as directed by policy rule 1 . This impacts the throughput offered to the Silver user bringing it to almost zero (this will be discussed in section C). Note that the rest of the Gold users (the ones that were already within range of BTS $A$ ) are unaffected. The three Gold users reach their throughput requirement (i.e. $128 \mathrm{Kbps}$ ) at simulation time 39 . The scheduling priority then shifts to the Silver users based on rule 2 since all the Gold users receive their throughput requirements. Taking the ischeduling priority away from the Gold users may drop their throughput below their required level. This shifts the scheduling priority once to the Gold users as dictated by rule 1 . Hence, the scheduling priority keeps switching between Gold and Silver users until the throughput requirements of the Silver users is met at time 63. This is illustrated in Figure 6 showing the throughput of the Gold users resembling a sinusoid between time 39 and 63. 
4) Scenario 4: Finally, we use the first simulation scenario to evaluate the fairness of our system. We calculate the standard deviation in the average throughput of all Gold and Silver users in the network separately. A fair scheduling algorithm should have a low value of standard deviation for both the Gold and Silver users. We compare against the QoS unaware scheduling algorithms. As expected, the results in Figure 7 (shown in logarithmic $\mathrm{y}$-axis scale) shows Max C/I to be the worst of all algorithm with standard deviation of about 212Kbits/sec for Gold users and 215Kbits/sec for Silver users. Our algorithms is shown to be the best of all 5 algorithms with a standard deviation of about $35 \mathrm{bits} / \mathrm{sec}$ for Silver users and $30 \mathrm{bits} / \mathrm{sec}$ for Gold users.

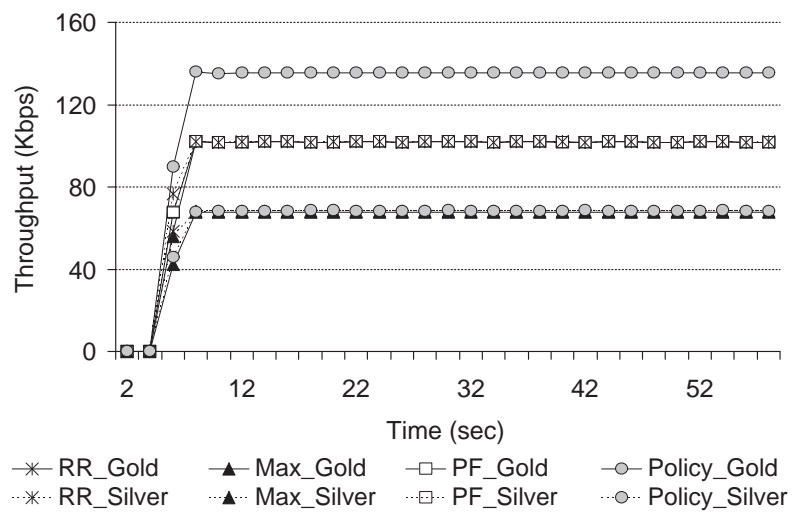

Fig. 4. Scenario One: A fixed topology with 12 Gold and 12 Silver users. Average throughput of Gold and Silver users are shown using different schedulers.

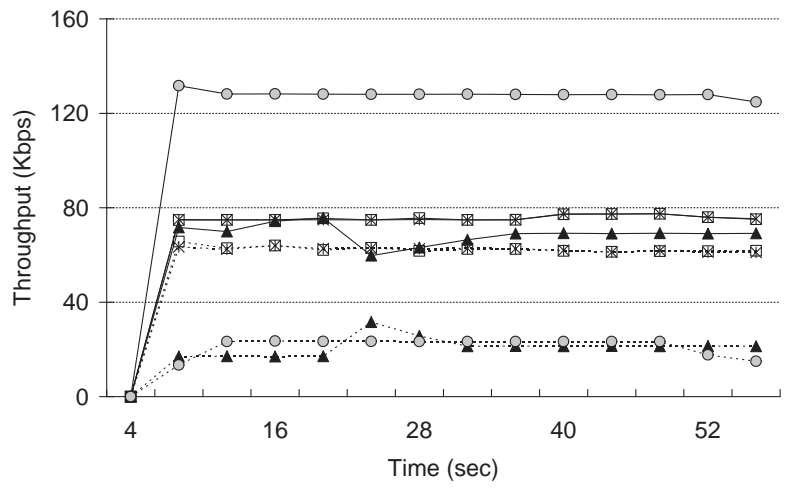

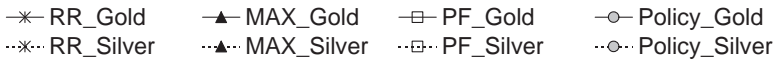

Fig. 5. Scenario Two: An overloaded network with 15 Gold and 15 Silver users. Average throughput of Gold and Silver users are shown using different schedulers.

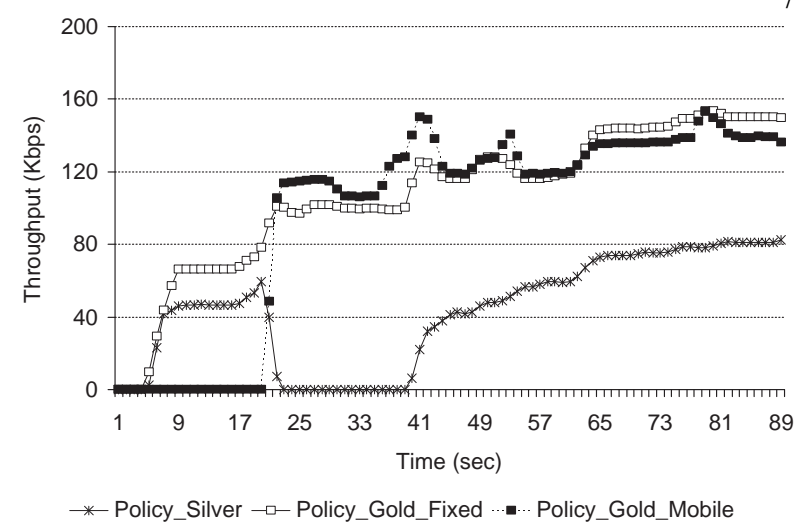

Fig. 6. Scenario Three: 3 mobile Gold users, 9 fixed Gold users and 12 fixed Silver users. Average throughput of fixed and mobile Gold users as well as that of the Silver users are shown using our policy based QoS scheduler.

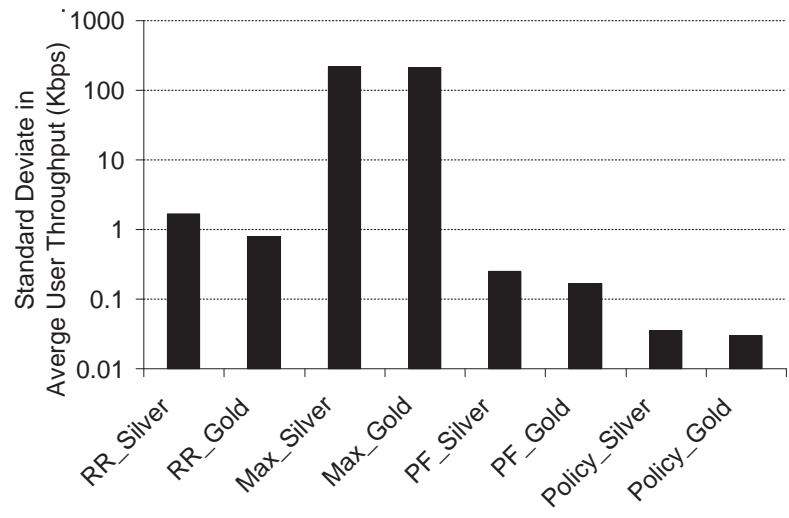

Fig. 7. Scenario Four: Illustrating fairness of our scheduler compared to that of other schedulers.

\section{Discussion}

In Figure 7, when Gold users move within range of BTS $A$ their $\mathrm{Ec} / \mathrm{Nt}$ is low. Hence, their data rate offered by the BTS will be low. It becomes impossible for the system to guarantee the Gold user's throughput requirements since the load offered by the BTS is lower than the required throughput. Hence, such users will monopolize the downlink transmission (along with the other Gold users that are not mobile) until their Ec/Nt matches their QoS requirements. Until then, the Silver users will always be of a lower scheduling priority which results in the deterioration of their throughput reaching zero between time 23 and 39 as shown from the Figure. If the Gold user was not mobile the network throughput could have been detrimentally impacted. This is an issue that has to be addressed by any QoS aware scheduler. 
A Gold user can clog the entire network if he stays within range of a BTS with Ec/Nt that does not match his throughput requirements. An extension to the policy is needed that would move such users to a lower QoS class that matches their current $\mathrm{Ec} / \mathrm{Nt}$.

In Figures 5 and 6, Max $\mathrm{C} / \mathrm{I}$ is shown to have a lower throughput compared to Round Robin and Proportional Fair. However, Max C/I has always been viewed as the scheduler that best utilizes the network resources achieving the highest throughput among other schedulers. Most evaluations of Max C/I assume that the queue of the user with the Max C/I is always full. If that is not the case, particulary when the queue has only a few packets, Max C/I performs poorly. Because of the good signal quality the user with the Max C/I has, he will be offered a physical layer packet size of up to 4096 bits as shown in Table 1. Our simulation results shows that if there is not enough packets in the queue to occupy the entire physical layer packet Max C/I results in poor utilization of network resources. This is a disadvantage of Max C/I schedulers especially that there may be other users with lower $\mathrm{C} / \mathrm{I}$ but a full queue that never get to be scheduled.

\section{CONCLUSiOnS}

In this paper we have introduced a QoS-aware policy-based scheduler for CDMA 1x Ev-DO. The scheduler takes into account the policy rules that govern the relationships between user QoS classes and follows these rules when selecting users to be served on the downlink. We implemented the CDMA 1x Ev-DO system in OPNET and evaluated it against MAX C/I, Round Robin, and Proportional Fair. The simulation results show that our scheduler performs well ensuring the policy rules are followed, meeting users' QoS requirements, and providing fairness among users within the same QoS class.

\section{REFERENCES}

[1] 3GPP2 C.S0024-0 v2.0, "cdma2000 High Rate Packet Data Air Interface Specification," 2000.

[2] 3GPP2 C.S0024-A v2.0, "cdma2000 High Rate Packet Data Air Interface Specification," 2005.

[3] 3GPP2 C.S0024-B v1.0, "cdma2000 High Rate Packet Data Air Interface Specification," 2006.

[4] OPNET Simulator, http://www.opnet.com/.

[5] Naga Bhushan, Chris Lott, Peter Black, Rashid Attar, Yu-Cheun Jou, Mingxi Fan, Donna Ghosh, Jean Au, "CDMA2000 1xEvDO Revision A: A Physical Layer and MAC Layer Overview," in IEEE Communication Magazine, 2006.
[6] Paul Bender, Peter Black, Matthew Grob, Roberto Padovani, Nagabhushana Sindhushayana, Andrew Viterbi, "CDMA/HDR: A Bandwidth-Efficient High-Speed Wireless Data Service for Nomadic Users," in IEEE Communication Magazine, 2000.

[7] Hossam Fattah, Cyril Leung, "An Overview of Scheduling Algorithms in Wireless Multimedia Networks," in IEEE Wireless Communications, 2002.

[8] Eun Ho Choi, Wan Choi, Jeffrey G. Andrews, "Throughput of the 1x Ev-DO System with Various Scheduling Algorithms," in Spread Spectrum Techniques and Applications, IEEE Eighth International Symposium on, 2004.

[9] Niranjan Joshi, Srinivas R. Kadaba, Sarvar Patel, Ganapathy S. Sundaram, "Downlink Scheduling in CDMA Data Networks," in MOBICOM, 2000.

[10] Cedric Westphal, "Monitoring Proportional Fairness in cdma2000 High Data Rate Networks," in IEEE GLOBECOM, 2004.

[11] Tian Bu, Li (Erran) Li, Ramachandran Ramjee, "Generalized Proportional Fair Scheduling in Third Generation Wireless Data Networks," in IEEE INFOCOM, 2006.

[12] A. Jalali, R. Padovani, R. Pankaj, "Data Throughput of CDMAHDR a High Efficient-High Data Rate Personal Communication Wireless System," in IEEE VTC, 2000.

[13] Jong Hun Rhee, Tae Hyung Kim, Dong Ku Kim, "A Wireless Fair Scheduling Algorithm for 1xEv-DO System," in IEEE VTC, 2001.

[14] Keivan Navaie, Halim Yanikomeroglu, "Downlink Joint Basestation Assignment and Packet Scheduling Algorithm for Cellular CDMA/TDMA Networks," in IEEE ICC, 2006.

[15] Patrick A. Hosein, "QoS Support on the 3G HRPD Air Interface," in IEEE Symposium on Wireless Personal Multimedia Communications, 2002.

[16] Young-June Choi, Saewong Bahk, "Scheduling for VoIP Service in cdma2000 1x Ev-DO," in IEEE Communications Society, 2004.

[17] Karina Gribanova, Riku Jantti, "On Scheduling Video Streaming Data in the HDR System," in IEEE VTC, 2004.

[18] Matthew Andrews, Krishnan Kumaran, Kavita Ramanan, Alexander Stolyar, Phil Whiting, "Providing Quality of Service over a Shared Wireless Link," in IEEE Communications Magazine, 2001.

[19] Kapseok Chang, Youngnam Han, "QoS-Based Adaptive Scheduling for a Mixed Service in HDR System," in IEEE PIMRC, 2002.

[20] Jian Song, Lemin Li, James J.Han, "A Wireless Fair Scheduling Algorithm Supporting CoS," in Communications, Circuits and Systems and West Sino Expositions, IEEE International Conference on, 2002.

[21] M. Andrews et al., "CDMA Data QoS Scheduling on the Forward Link with Variable Channel Conditions," in Bell Labs Tech. Memo., 2000.

[22] S. Shakkottai, A. Stolyar, "A Study of Scheduling Algorithms for a Mixture of Real- and Non-Real-Time Data in HDR," in Bell Labs Tech. Memo., 2000.

[23] Sborst, P. Whiting, "Dynamic Rate Control Algorithms for HDR Throughput Optimization," in IEEE INFOCOM, 2001.

[24] A. Jalali, R. Padovani, and R. Pankaj, "Data throughput of CDMAHDR a high efficiency-high data rate personal communication wireless system," in VTC, 2000.

[25] Robert C. Elliott and Witold A. Krzymien, "Scheduling Algorithms for the cdma2000 Packet Data," in VTC, 2002. 